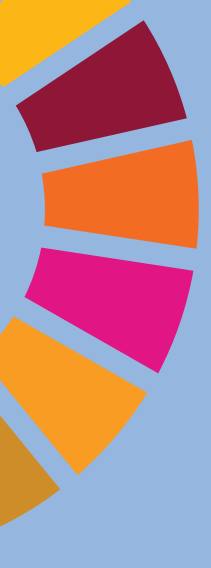

\title{
LA ISR EN ESPAÑA: CLAVES, TENDENCIAS Y PREVISIONES DESDE SPAINSIF
}

\section{SRI IN SPAIN: DRIVERS, TRENDS AND FORECASTS FROM SPAINSIF}

\author{
Andrea González González \\ Spainsif, Foro español de la Inversión Sostenible \\ andreagonzalez@spainsif.es \\ Verónica Sanz Izquierdo \\ Spainsif, Foro español de la Inversión Sostenible \\ vsanz@spainsif.es
}

\section{RESUMEN}

Durante los últimos años, la inversión sostenible y responsable en España ha crecido no solo en el patrimonio de los activos gestionados con criterios Ambientales, Sociales y de Gobernanza, sino en la sofisticación de las estrategias de selección, integración e influencia empleadas. El extendido uso del marco de los Objetivos de Desarrollo Sostenible de Naciones Unidas, el crecimiento de los mercados de bonos verdes y sociales, así como el impulso regulatorio europeo a las finanzas sostenibles, son los ejes de expansión de las finanzas sostenibles en el presente. Por el lado de las tendencias, el impulso de la financiación climática en la recuperación económica a la crisis COVID-19, la evolución del mercado de la inversión sostenible en América Latina y la calidad y disponibilidad de información no financiera serán clave en el desarrollo de esta filosofía de inversión en el futuro próximo.

Palabras clave: Inversión Sostenible y Responsable; Objetivos de Desarrollo Sostenible; Aspectos Ambientales; Aspectos sociales; Cambio Climático; Información No Financiera; COVID-19; Recuperación; Inversión de Impacto; Bonos Verdes; Bonos Sociales. 


\section{ABSTRACT}

In recent years, sustainable and responsible investment in Spain has grown not only in the quantity of assets managed under Environmental, Social and Governance criteria, but in the sophistication of the selection, integration and influence strategies applied. The widespread use of the United Nations Sustainable Development Goals framework, the growth of green and social bond markets, as well as the European regulatory momentum for sustainable finance, are the paths for expansion of sustainable finance in present. Regarding trends, the promotion of climate finance in the economic recovery from the COVID-19 crisis, the sustainable investment market evolution in Latin America and the quality and availability of non-financial information will be key in the development of this investment philosophy in the near future.

Keywords: Sustainable and Responsible Investment; Sustainable Development Goals; Environmental criterion; Social criterion; Climate Change; Non-Financial Information; COVID-19; Recovery; Impact Investing; Green Bonds; Social Bonds.

Andrea González González. Economista especializada en sostenibilidad y responsabilidad social corporativa, ha desarrollado parte de su trayectoria profesional en organizaciones internacionales como ONU-OCHA, la OMS o el Parlamento Europeo. Es colaboradora habitual en centros de educación superior como la Universidad Internacional Menéndez Pelayo o la Universidad Carlos III de Madrid. En el presente forma parte del equipo de Spainsif, foro español de inversión sostenible, donde ocupa el puesto de subdirectora general.

Verónica Sanz Izquierdo. Licenciada en Ciencias Ambientales, cuenta con varios postgrados relacionados con la auditoría y verificación ambiental y social, así como con geopolítica y gobernanza. Previamente ha trabajado en el sector privado en consultoría ambiental y en asociaciones y corporaciones de derecho público profesionales. Actualmente es Responsable de Análisis y Estudios en Spainsif, donde realiza estudios y coordina la sección de formación sobre la inversión con criterios ambientales, sociales y de buen gobierno en el mercado español, así como las iniciativas y regulaciones aplicables.

Spainsif, el Foro Español de la Inversión Sostenible y Responsable (en adelante, ISR) monitoriza desde 2009 la evolución de este tipo de inversión en España, en el marco de la metodología establecida por Eurosif, asociación para la promoción y el avance de la inclusión de los criterios ambientales, sociales y de buena gobernanza (ASG) en las políticas de inversión en Europa.

La definición de inversión sostenible y responsable (ISR) varía ligeramente a lo largo de la literatura, en función de las atribuciones de las entidades que la emita. Como referencia, según la Comisión Europea, la Inversión Sostenible y Responsable en el Reglamento 2019/2088 de la UE sobre divulgaciones relacionadas con la sostenibilidad en el sector de servicios financieros se describe como "Una inversión en una actividad económica que contribuya a un objetivo ambiental, o una inversión en una actividad económica que contribuya a un objetivo social, siempre que dichas inversiones no perjudiquen significativamente ninguno de esos objetivos y que las sociedades participadas sigan prácticas de buen gobierno" (Comisión Europea, 2018). Es decir, se establece como ISR a aquellas inversiones que incorporan criterios ambientales, sociales y de buen gobierno (ASG, o ESG por sus siglas en inglés) en el proceso de estudio, análisis y selección de valores de una cartera de inversión. 
La aplicación de estos criterios varía en función de la entidad que ejecute la inversión, empleándose el uso de una o varias estrategias que, con distinto grado de sofisticación, permiten identificar aquellos activos alineados con la política de inversión establecida. Como estrategias más sencillas se establecen aquellas basadas en la selección de valores vía exclusiones o cribados previos, como son las exclusiones simples o el screening basado en normas. Como estrategias de mayor calidad y alto grado de análisis se encuentra la integración ASG o la inversión de impacto. Habitualmente, la cartera es resultado de la aplicación de varias estrategias sobre un mismo universo.

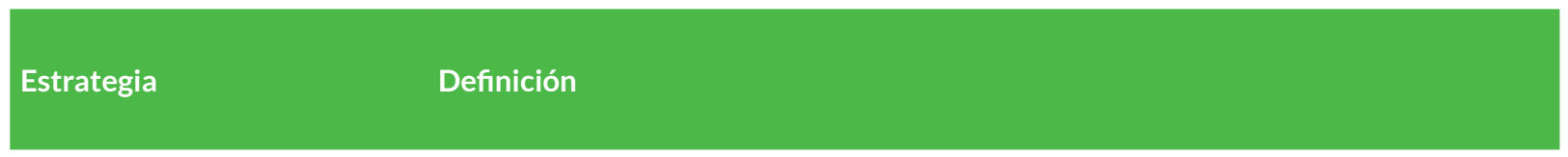

Exclusión Excluye ciertas actividades o sectores, por ser contrarios a los valores del inversor

Best-in Class Da prioridad a las empresas mejor valoradas desde la perspectiva extra-financiera, dentro de su sector de actividad.

Screening basado en normas Selección de organizaciones a través de normas y convenciones internacionales.

Fondos temáticos Selecciona empresas e inversiones vinculadas a temáticas o sectores de desarrollo sostenible.

Engagement and Voting Diálogo directo entre el inversor y las organizaciones donde invierte, en materia ASG. Si el diálogo no fructifica utilizan su derecho a voto en las juntas de accionistas.

Integración de criterios ASG Consiste en tener en cuenta en la gestión clásica de inversiones criterios ASG.

Pretende simultáneamente obtener un rendimiento financiero competitivo y

Inversión de Impacto producir un impacto ambiental y/o social positivo. La medición del impacto debe ser cuantificable, con el fin de lograr cambios significativos en la resolución de los problemas sociales y/o ambientales.

Según el estudio de Spainsif de 2020, resultado del análisis de los datos reportados por 33 entidades, 21 de ellas nacionales y 12 gestores y propietarios de activos internacionales, lo que supone el $76 \%$ del total del nacional y un $43 \%$ del internacional comercializado en España, los activos gestionados con algún tipo de estrategia o criterio ambiental, social y de gobernanza ASG, ascienden a un total de 285.454 millones de euros. 
Ilustración 1. Evolución de las diferentes estrategias ASG en activos nacionales durante los últimos diez años. Fuente: Spainsif a partir de los datos recibidos en las encuestas.

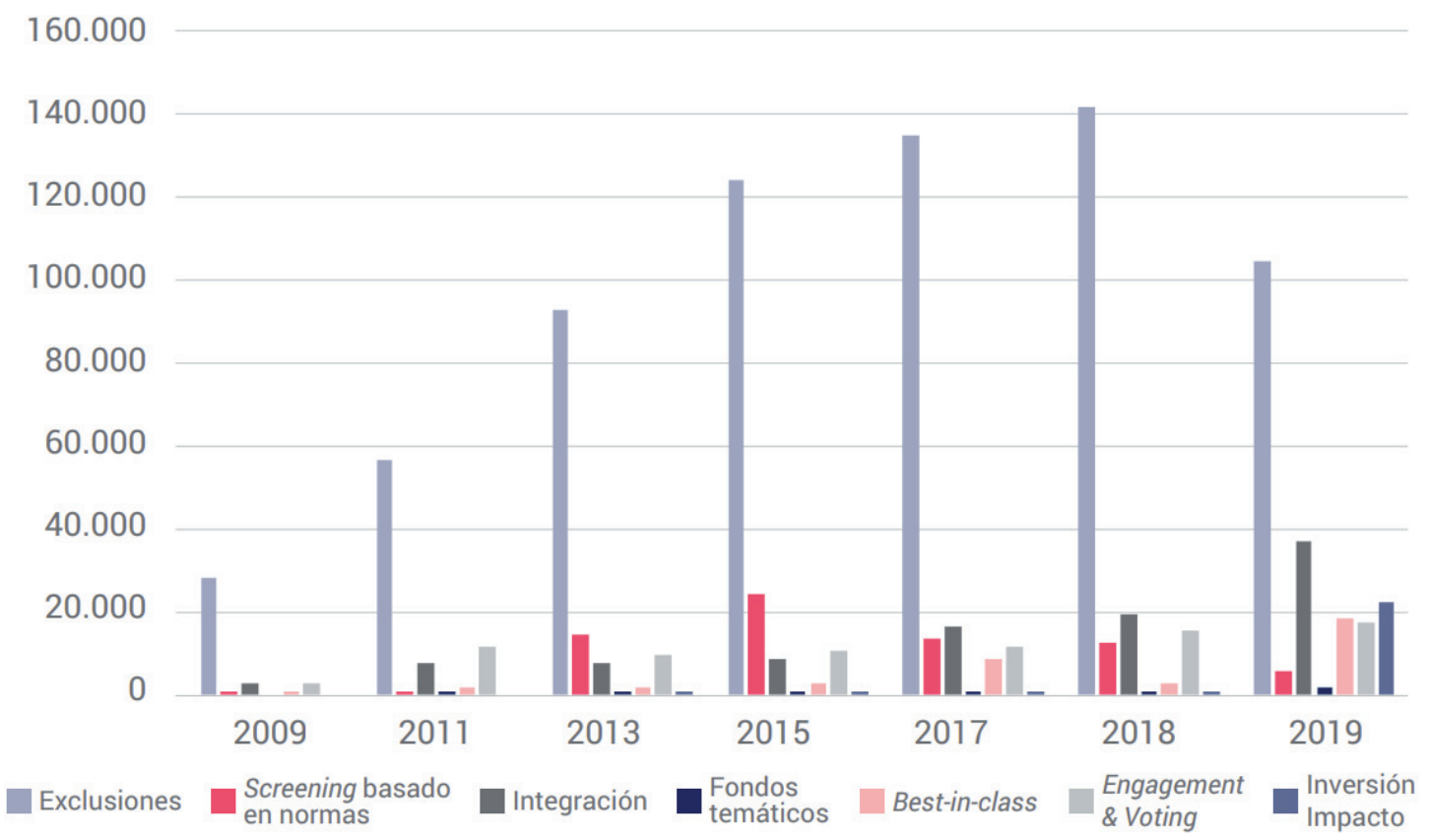

Atendiendo al reparto por estrategias (Ilustración 1), que es indicador del grado de madurez y calidad de la ISR, destaca el crecimiento que presenta la integración ASG, que alcanza los 100.189 millones de euros en 2019, señalada como una de las estrategias más sofisticadas en el marco metodológico de Eurosif; si bien la exclusión simple continúa siendo la estrategia más aplicada en España sumando 104.245 millones de euros en 2019.

Con respecto a las inversiones temáticas sostenibles, que suponen según los datos recabados en este estudio, un total de 2.530 millones de euros en España (un 1\% del total ASG), destaca la mayor propensión de las entidades internacionales a desarrollar este tipo de estrategia, en un contexto donde el mercado nacional es todavía reducido y concentrado en torno a sectores de corte ambiental como las energías renovables, la eficiencia energética y la gestión del agua.

Destaca también el crecimiento que han evidenciado las inversiones de impacto, que suponen 29.567 millones de euros y una décima parte del total, relacionadas con los Objetivos de Desarrollo Sostenible (ODS), los bonos verdes y sociales, y los fondos de inversión principalmente. Además, el 10\% de las inversiones de impacto monitorizadas en el estudio tienen como objetivo contribuir a alcanzar los Objetivos de Desarrollo Sostenible (en adelante, ODS).

Durante 2019, las emisiones de bonos verdes y bonos sostenibles crecieron de forma destacada, lo cual podría explicar, al menos en parte, el aumento en las cifras de activos gestionados bajo la estrategia de inversión de impacto en España. Desglosando la información por el origen de las organizaciones, se aprecian ligeras diferencias: el porcentaje de entidades nacionales con inversión de impacto en fondos de inversión, capital riesgo social e inversión en microfinanzas y bonos sociales supera al de internacionales; por el contrario, el número de entidades internacionales con inversiones en bonos verdes y ODS superan a las nacionales. 
Con respecto al engagement, más de la mitad de las empresas encuestadas declara disponer de una política formal y en la misma relación hace públicos los resultados de sus campañas. Sobre el voting, dos tercios de los encuestados cuentan con una política formal y casi la mitad de ellos publica el sentido de sus votaciones. Estas estrategias alcanzaron los 21.059 millones de euros según los datos del estudio.

La rápida evolución de la ISR y la proliferación de una gran cantidad de tipologías de productos bajo los epígrafes de sostenibles, verdes o responsables, con desigual grado de interiorización de los aspectos sociales, ambientales o de gobernanza, ha incentivado la necesidad de generar un marco en el que se definan y concreten los conceptos, actores y responsabilidades a la hora de gestionar los criterios ASG en el sector financiero.

Por ello, en 2018 la Comisión Europea diseñó y puso en marcha un Plan de Acción en Finanzas Sostenibles en el que destacan acciones como la elaboración de la Taxonomía de actividades verdes, en la que se establece los criterios para determinar si una actividad económica se considera medioambientalmente sostenible a efectos de fijar el grado de sostenibilidad ambiental de una inversión; el Estándar de Bono Verde Europeo, que pretende facilitar el desarrollo del mercado al unificar las metodologías y mejorar la transparencia en la gestión; y la creación de dos índices de referencia climáticos, para fomentar la comparabilidad y aumentar la transparencia del impacto de las inversiones en materia de cambio climático y transición energética, y desincentivar el greenwashing. Este marco regulatorio europeo en finanzas sostenibles, junto con la demanda de los inversores individuales, se señalan en el estudio anual de Spainsif presentado en 2020 como dos de los factores clave para el desarrollo de la ISR en los próximos años.

Como novedad, en el Estudio de 2020 se recoge una primera aproximación a las finanzas sostenibles en América Latina, en colaboración con Principles for Responsible Investment (PRI), a través de una muestra de signatarios de la región. Según los datos analizados, entre los que se excluye Brasil por la singularidad de su mercado, la actividad de la inversión sostenible en América Latina se centra principalmente en Chile, Colombia, México y Perú; donde se concentran los inversores institucionales de mayor peso de la región: los fondos privados de pensiones.

Todas las entidades encuestadas cuentan con una política definida de integración ASG, una estrategia considerada en los mercados europeos como una de las de mayor calidad a la hora de afrontar la inversión responsable. Este hecho se debe en gran medida a la labor de comunicación y divulgación realizada por PRI. Como contrapartida, algunos datos indican que no es posible aplicar estrategias de exclusión tanto positivas como negativas, debido al tamaño tan reducido del universo invertible con el que cuentan.

Al encuestar a los participantes en el estudio sobre las temáticas incluidas en sus estrategias en relación con el desarrollo sostenible y el cambio climático, destacan por encima de las demás los Objetivos de Desarrollo Sostenible. Estos objetivos reflejan las inquietudes de las sociedades de América Latina, muy afectadas por problemas como el hambre, el nivel educativo o la desigualdad de género. Por ello, es frecuente encontrar entidades comprometidas profundamente con ellos.

También durante 2020 se publicó el Estudio temático de Spainsif "Las Inversiones y el Cambio Climático", donde se ponía el foco en el papel de las finanzas ante el reto de la Emergencia Climática. El estudio recopila información sobre el cambio climático en las finanzas a través de una revisión bibliográfica y la realización de encuestas y entrevistas a agentes clave del sector.

Las percepciones de los agentes implicados en el estudio identifican los riesgos de transición como los más relevantes para el sector, destacando los riesgos de mercado y derivados de las políticas públicas. El sector financiero igualmente considera que los riesgos físicos, en concreto los crónicos, como el aumento de las temperaturas o la escasez de recursos tendrán efectos relevantes sobre el sistema financiero. 
Los agentes entrevistados destacan las numerosas oportunidades de inversión que ofrecen tanto la adaptación como la mitigación del cambio climático en sectores como las energías renovables, especialmente la energía solar, junto a la eficiencia energética, y evidencian que el mercado todavía debe adquirir experiencia y formación a este respecto, espoleado por la demanda de los inversores, especialmente institucionales, y las reformas regulatorias.

Según las respuestas recibidas, se evidencia un aumento del peso relativo de las inversiones climáticas en las carteras de inversión. En cuanto a los instrumentos financieros, los bonos verdes y los bonos sostenibles, aquellos que además de aspectos ambientales tienen en cuenta aspectos sociales, son percibidos como los instrumentos con mayor potencial para el futuro próximo. Destaca que dos tercios de los encuestados creen que habrá un aumento de la financiación climática como consecuencia de la crisis del COVID-19, sea por el crecimiento generalizado de la financiación sostenible o gracias a planes verdes de estímulo económico; e identifica el papel del nuevo marco jurídico europeo como impulsor de las finanzas sostenibles climáticas.

Dada la situación excepcional de emergencia social provocada por el COVID-19, se publicó durante 2020 un tercer estudio temático de Spainsif, centrado en los aspectos sociales de la inversión sostenible. Tradicionalmente la parte ambiental ha supuesto la mayor ratio de la inversión sostenible, mientras que los aspectos sociales se relegaban al cumplimiento de normativa básica o a unos mínimos acordes con el derecho internacional. Sin embargo, se ha puesto de manifiesto una relevancia creciente de estos temas en los últimos años, fuertemente impulsada en los últimos meses por la emergencia sanitaria.

Como impulsores de los componentes sociales en la inversión sostenible, destacan por encima de otros, la contribución de los ODS de la Agenda 2030 de Naciones Unidas, con un fuerte componente social, incentivado por las desigualdades y carencias existentes a nivel mundial. Entre los objetivos y metas planteados se recogen: poner fin a la pobreza, como objetivo número uno, acabar con el hambre y garantizar la seguridad alimentaria, en segundo lugar, la salud y bienestar como tercer objetivo, seguidos de alcanzar una educación de calidad, igualdad de género y empoderamiento de la mujer, abastecimiento de agua y saneamiento, reducir las desigualdades tanto entre países como dentro de los mismos, y garantizar la paz y la justicia.

Se identificó como especialmente relevante para las finanzas sostenibles el ODS 8 de "Trabajo decente y crecimiento económico", que recoge entre sus metas "lograr el empleo pleno y productivo y el trabajo decente para todas las mujeres y los hombres, incluidos los jóvenes y las personas con discapacidad, así como la igualdad de remuneración por trabajo de igual valor" o la adopción de "medidas inmediatas y eficaces para erradicar el trabajo forzoso y poner fin al trabajo infantil en todas sus formas en 2025", sin perder el foco en otras metas financieras como "Mantener el crecimiento económico per cápita, de conformidad con las circunstancias nacionales $y$, en particular, un crecimiento del producto interno bruto de al menos el $7 \%$ anual en los países menos adelantados" o "Fortalecer la capacidad de las instituciones financieras nacionales para fomentar y ampliar el acceso a los servicios bancarios, financieros y de seguros para todos".

Los ODS son frecuentemente utilizados como referencia para los reportes de sostenibilidad; sin embargo, es difícil cuantificar la aportación de las entidades al logro de estos objetivos globales y no existe un sistema unificado de divulgación en base a ellos. Desde el enfoque del inversor ASG, los reportes de sostenibilidad de las empresas respecto a los ODS suelen ser complementados y ajustados a los propios indicadores de inversión ASG, atendiendo al mandato fiduciario del inversor institucional. Para ello, los inversores ASG cuentan con proveedores de calificación y rating especializados, que aplican su propia aproximación cuantitativa a los aspectos sociales relacionados con cada ODS (incluidos en el diseño del vehículo de inversión del que se trate). 
En cuanto al marco regulatorio europeo, destaca la Directiva de Información No Financiera, traspuesta a la legislación española en 2018, que presta especial atención a los aspectos sociales de diversidad, género, distribución del trabajo, relaciones con partes interesadas y efectos sobre la comunidad, de los que tienen ya obligación de reportar las empresas a partir de un tamaño determinado. Aunque no existe una metodología unificada de reporting, y eso complica la comparabilidad a la vez que genera confusión, es reseñable la aportación de las principales iniciativas internacionales de uso voluntario en la materia, como son Global Reporting Initiative (GRI), Sustainability Accounting Standards Board (SASB) o Integrated Report (IR), que incorporan específicamente los aspectos sociales.

Contemplando las iniciativas relacionadas con la transición ecológica, incentivada por el Pacto Verde Europeo, se establecen mecanismos para que la transición sea justa; es decir, que no deje a nadie atrás y que mitigue los impactos negativos que la descarbonización pueda tener en los distintos sectores y regiones. En el caso de España, el proyecto de Ley del Clima recoge un Título específico dedicado a este fin. A falta de una taxonomía social, demanda escuchada por los reguladores, que han comenzado a trabajar en ella, en la taxonomía verde se recogen aquellas actividades económicas que tienen contribución sustancial a los objetivos ambientales de la UE y se obliga a demostrar el cumplimiento de unas garantías sociales mínimas a través de la alineación con directrices y normativa internacional en materia de trabajo, asociación y derechos humanos.

Si ya en la situación inicial de shock y contracción del mercado financiero provocada por la irrupción de la pandemia del COVID-19 la inversión sostenible evidenció mejores comportamientos que la tradicional, para la recuperación económica posterior las finanzas sostenibles se recogen como pieza clave, no solo en cuestiones sociales, como las perseguidas en la emisión de bonos sociales o bonos COVID-19, si no a través del programa Next Generation EU y los fondos de recuperación, con los que se pretende restaurar la economía sentando las bases de un nuevo desarrollo económico alineado con los objetivos ambientales de la Unión Europea. 


\section{BIBLIOGRAFÍA}

- Comisión Europea. (2018). Comunicación de la Comisión Europea, Plan de Acción: Financiar el desarrollo sostenible. COM (2018) 97 final. Bruselas, 8.3.2018. Recuperado de: https://eur-lex.europa.eu/legalcontent/ES/TXT/HTML/?uri=CELEX:52018DC0097\&from=EN_blank

- Spainsif, Foro Español de Inversión Sostenible y Responsable. (2020). Estudio Spainsif 2020: La inversión sostenible y responsable en España. Recuperado de: https://www.spainsif.es/af_estudio_anual_ spainsif_2020web-2/

- Spainsif, Foro Español de Inversión Sostenible y Responsable. (2020). Las inversiones y el cambio climático. https://www.spainsif.es/estudio-spainsif-cambio-climatico/

- Spainsif, Foro Español de Inversión Sostenible y Responsable. (2020). La dimensión social de la inversión sostenible. Recuperado de: https://www.spainsif.es/wp-content/uploads/2019/10/Estudio-2019.pdf 